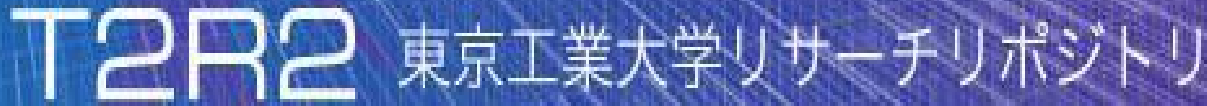

\section{Tokyo Tech Research Repository}

\section{論文 /著書情報 \\ Article /Book Information}

\begin{tabular}{|c|c|}
\hline Title & The Reduction of Dumping Factor at Well-in-Well Quantum Well Lasers \\
\hline Author & $\begin{array}{l}\text { Yasutaka Higa, Mikio Sorimachi, Takuya Nishinome, Hajime Iwasaki, } \\
\text { A kihiro Matsutani, Tomoyuki Miyamoto }\end{array}$ \\
\hline Journal/Book name & IEEE PHOTONICS 2011, Vol. , No. , pp. 680-681 \\
\hline Issue date & 2011, 10 \\
\hline DOI & http://dx.doi.org/10.1109/PHO .2011.6110732 \\
\hline URL & http://www.ieee.org/ndex.html \\
\hline Copyright & $\begin{array}{l}\text { (c) } 2011 \text { IEEE. Personal use of this material is permitted. Permission } \\
\text { from IEEE must be obtained for all other users, including } \\
\text { reprinting/republishing this material for advertising or promotional } \\
\text { purposes, creating new collective works for resale or redistribution to } \\
\text { servers or lists, or reuse of any copyrighted components of this work in } \\
\text { other works. }\end{array}$ \\
\hline Note & $\begin{array}{l}\text { このファイルは著者 (最終) 版です。 } \\
\text { This file is author (final) version. }\end{array}$ \\
\hline
\end{tabular}




\title{
The Reduction of Dumping Factor at Well-in-Well Quantum Well Lasers
}

\author{
Yasutaka Higa, Mikio Sorimachi, Takuya Nishinome, Hajime Iwasaki, and Tomoyuki Miyamoto \\ Photonics Integration System Research Center, $P$ \& I Lab., Tokyo Institute of Technology. \\ R2-39, 4259 Nagatsuta, Midoriku, Yokohama 226-8503, Japan \\ e-mail address: yasutaka.higa@ms.pi.titech.ac.jp
}

\begin{abstract}
A quantum well design for controlling of the carrier relaxation process and reducing of the gain damping effect was investigated for improvement of high speed direct modulation semiconductor lasers. The proposed Well-in-Well structure decreases the carrier relaxation time into an active well by carefully designed wave function which relates to the $\mathrm{LO}$ phonon scattering rate. The decrease of relaxation time and the increase of modulation bandwidth was analyzed numerically. The reduction of damping factor $\gamma$ was also demonstrated by experimental measurement of the relative intensity noise spectrum.
\end{abstract}

\section{INTRODUCTION}

Quantum well (QW) lasers have been progressed and the direct modulation bandwidth has become fast up over $20 \mathrm{GHz}$. However, increase of the modulation bandwidth by structural optimization methods is limited by the damping effect of the optical gain. To suppress the dumping effect due to the finite carrier relaxation time, a tunnel injection quantum well (TIQW) structure (Fig. 1(a)) was proposed [1,2]. The TIQW structure can control the carrier relaxation time into an active well by designing the wave function which relates to the LO-phonon scattering.

In this paper, a Well-in-Well (WWell) structure is proposed for controlling the carrier relaxation time and dumping effect to improve the modulation bandwidth. The WWell has the same elemental functions as the TIQW and a simple layer structure. It is noted the WWell is proposed to increase the LO phonon scattering by designing the quantum structure in detail and is different from the conventional GRIN-SCH which has similar potential steps. The electron relaxation characteristics dependence on the structure was analyzed numerically and the MBE-grown device was tested for clarifying the reduction of the damping effect via the RIN spectrum measurement.

\section{CONCEPT AND ANALYSIS OF WWELL}

Figure 1 shows a schematic model of conduction band diagram and electron wave functions of a single WWell

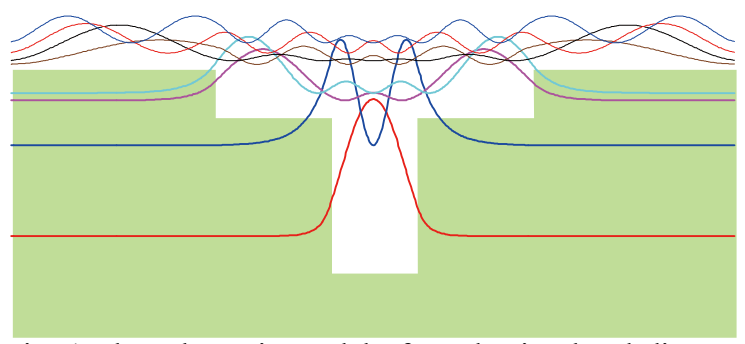

Fig. 1 The schematic model of conduction band diagram and electron wave functions of WWell structure.

structure. The WWell structure is composed of an inner active well that is sandwiched by outer wells. At the WWell structure, outer well structure controls the wave functions and makes large overlap of wave functions between the barrier region and itself, and also between an active well and itself. This large overlap leads a large scattering rate of electrons by LO phonon. Because the LO phonon scattering causes energy relaxation of the carriers, electron relaxation time is decreased by controlling the wave functions.

Firstly, the theoretical analysis based on this LO phonon scattering rate was investigated. We composed the analysis model for this simulation, and investigated the carrier relaxation time and modulation bandwidth [3].

Figure 2 shows the dependence of electron relaxation time on outer well width at WWell structure. In the numerical analysis, the barrier or SCH layer thickness of one side was assumed to be $30 \mathrm{~nm}$. It is noted that the structures whose outer well width was 0 $\mathrm{nm}$ and $30 \mathrm{~nm}$ correspond to the conventional QW structures of shallow and deep well depth, respectively. The relaxation time is reduced most when the outer well width is approximately $10 \mathrm{~nm}$. The minimum relaxation time was $3.7 \mathrm{ps}$ and corresponding $\mathrm{K}$-factor was $0.15 \mathrm{~ns}$ at typical laser parameter. At conventional InGaAs/GaAs QW laser for high speed direct modulation, reported K-factor was $0.24 \sim 0.29$ ns [4,5]. Comparing with these values, we think that the $\mathrm{K}$ factor can be reduced by the WWell structure. 


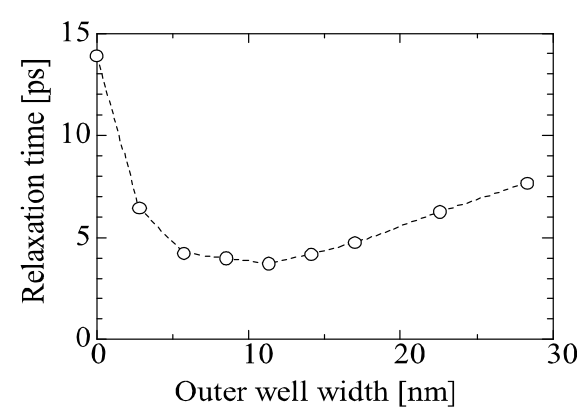

Fig. 2 Calculated relaxation time dependence on the outer well width of WWell structure.

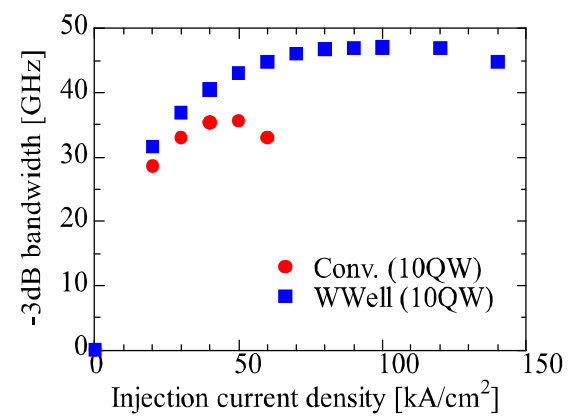

Fig. 3 Theoretical $-3 \mathrm{~dB}$ bandwidth of $10 \mathrm{QW}$ lasers.

Figure 3 indicates the calculated $-3 \mathrm{~dB}$ bandwidth of conventional QW and WWell lasers. The WWell laser structure was optimized to the fastest electron relaxation. The active region of both lasers was composed by 10QW and photon lifetime was set to be 1.3 ps. The result shown in Fig. 3 suggested that the WWell structure can expand the $-3 \mathrm{~dB}$ bandwidth because of the faster carrier relaxation. As shown in Fig. 3, the maximum bandwidth is expected to be increased from approximately $35 \mathrm{GHz}$ to $50 \mathrm{GHz}$.

\section{MESUREMTN OF RERATIVE INTENSITY NOISE}

A WWell laser wafer was grown by MBE system, and fabricated to ridge lasers for characterization of the relative intensity noise (RIN) characteristics. We prepared $\mathrm{Ga}_{0.8} \mathrm{In}_{0.2} \mathrm{As} / \mathrm{GaAs}$ conventional $3 \mathrm{QW}$ lasers and $\mathrm{Ga}_{0.8} \mathrm{In}_{0.2} \mathrm{As} / \mathrm{GaAs} / \mathrm{Al}_{0.05} \mathrm{Ga}_{0.95} \mathrm{As}$ WWell $3 \mathrm{QW}$

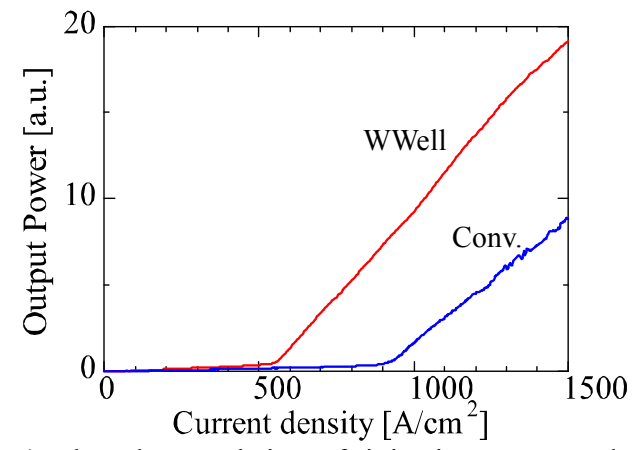

Fig. 4 The characteristics of injection current density versus output power of $4 \mu \mathrm{m} \times 500 \mu \mathrm{m}$ ridge lasers.

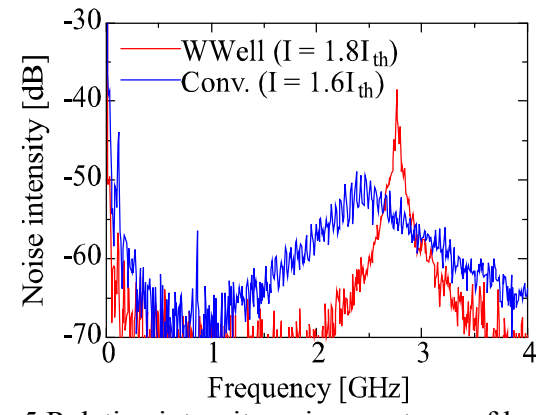

Fig. 5 Relative intensity noise spectrum of lasers.

lasers. Figure 4 shows the current density versus output power characteristics of these lasers. The difference of threshold current density is explained by the small fabrication error of the cavity width and the photon lifetime.

Figure 5 shows the result of RIN spectrum measurement. The peak of RIN spectrum of the WWell structure was larger than that of the conventional QW. This difference indicates that the damping factor of the WWell laser is smaller than that of the conventional QW laser from the theoretical relation shown below:

$$
\frac{\mathrm{RIN}}{\Delta f}=\frac{4 \Gamma R_{s p}^{\prime}}{N_{p}} \cdot \frac{1}{\gamma^{2}}
$$

It is noted that the difference of photon density is not enough to explain the $10 \mathrm{~dB}$ difference of RIN peaks.

In conclusion, a novel quantum well active region "WWell" was proposed to reduce the electron relaxation time. By theoretical analysis, the shortening effect of electron relaxation and expanding effect of direct modulation bandwidth were studied. The reduction effect of damping factor was demonstrated by measurement of the RIN peak intensity. The WWell is advantageous to improve the direct modulation response of the semiconductor lasers by suppression of the gain damping.

[1] H. C. Sun, L. Davis, S. Sethi, J. Singh, and P. Bhattacharya, "Properties of a Tunneling Injection Quantum-Well Laser: Recipe for a "Cold"Device with a Large Modulation Bandwidth", IEEE Photon. Technol. Lett. 5, 870 (1993).

[2] Yasutaka Higa, Tomoyuki Miyamoto, Hiroshi Nakajima, Kosuke Fujimoto, and Fumio Koyama, "Theoretical design of carrier injection rate and recombination rate in tunnel injection quantum well lasers", Phys. Stat. Sol. (c) 5, 2838 (2008).

[3]Yasutaka Higa, Mikio Sorimachi, Takuya Nishinome, and Tomoyuki Miyamoto , " Control of carrier relaxation for suppression of optical gain damping by using Well-in-Well structure ", NUSOD 2011, WD5

[4] John D. Ralston, Stefan Weisser, Ignacio Esquivias, Eric C. Larkins, Josef Rosenzweig, Paul J. Tasker, and Joachim Fleissner, "Control of Differential Gain, Nonlinear Gain, and Damping Factor for High-speed Application of GaAs-Based MQW Lasers", IEEE, J. Quantum Electron. 29, 1648 (1993)

[5] S. Weisser, E. C. Larkins, K. Czotscher, W. Benz, J. Daleiden, I. Esquivias, J. Fleissner, J. D. Ralston, B. Romero, R. E. Sah, A. Schonfelder, and J. Rosenzweig, "Damping-Limited Modulation Bandwidths Up to $40 \mathrm{GHz}$ in Undoped Short-Cavity $\mathrm{In}_{0.35} \mathrm{Ga}_{0.65}$ AsGaAs Multiple-Quantum-Well Lasers", IEEE Photon. Technol. Lett. 8, 608 (1996). 